\title{
INFLUÊNCIA DA FISIOTERAPIA NA FORÇA MUSCULAR RESPIRATÓRIA E FORÇA DE PREENSÃO PALMAR DE PACIENTES HOSPITALIZADOS
}

Guilherme Henrique Dalaqua Grande, Layane Lopes Napoleão, Fabiano Francisco de Lima, Caroline

Braun, Tamara Caroline Barbosa, Susimary Aparecida Trevizan Padulla

Universidade Estadual Paulista - FCT/UNESP. Presidente Prudente, SP. e-mail: susi@fct.unesp.br

\section{RESUMO}

Ensaio clínico com características prospectivas com intervenção fisioterápica. Tem como objetivo caracterizar o perfil dos pacientes internados na Santa Casa de Presidente Prudente encaminhados à Fisioterapia e avaliar o efeito da fisioterapia sobre a Pressão Inspiratória máxima (Pimax), Pressão Expiratória máxima (PEmax) e força de preensão palmar (FPP). Participaram 93 indivíduos internados que realizavam fisioterapia. Cada voluntário foi questionado no leito e submetido ao teste de dinamometria para avaliar a força de preensão palmar e ao teste de manovacuometria, para obtenção das pressões inspiratória e expiratória máximas. Utilizou-se estatística descritiva, teste qui-quadrado, t-student, e correlação de pearson, utilizando o programa BioEstat 5.0. A causa de internação predominate foi o câncer e o fator de risco mais prevalente foi o sedenterismo. Quanto as variáveis Pimax, Pemax e FPP, não houve diminuição significativa, considerando o tempo de internação. Não houve alterações na força muscular respiratória e na preensão palmar durante o período de internação nos pacientes que realizavam a fisioterapia, sendo esta capaz de prevenir as alterações funcionais que são impostas ao paciente como efeito do imobilismo e restrição ao leito.

Palavras-chave: Pacientes hospitalizados, Fisioterapia, Força muscular.

\section{INFLUENCE OF PHYSICAL THERAPY IN RESPIRATORY MUSCLE STRENGTH AND POWER HOLD OF PATIENTS HOSPITALIZED PALMAR}

\begin{abstract}
A clinical essay with prospective characteristics in which there was no intervention. Their objective characterizing profiles of patients admitted to Santa Casa de Presidente Prudente referred for taking physical therapy and to evaluate the effect of therapy on maximal inspiratory pressure (MIP), maximal expiratory pressure (MEP) and palmar prehension (FPP). There were 93 individuals admitted to undergo physiotherapy. Each volunteer was asked in bed and tested for dynamometry to assess a palmar prehension test and manometer to obtain the maximum inspiratory and expiratory pressures. It was used descriptive statistics, qui-square, t-student, and pearson correlation, using the BioEstat 5.0. program. Cancer was the predominating cause of hospitalization and the most prevalent risk factor was sedentary lifestyle. About MIP, MEP and FPP there was no significant decrease considering the time of admission. There were no changes in respiratory muscular strength and palmar prehension during the time spent in hospital for patients who underwent physiotherapy, which is capable of preventing functional alterations that are imposed to the patient as an effect of immobility and bed restriction.
\end{abstract}

Keywords: Hospitalized patients, physiotherapy, muscle strength. 


\section{INTRODUÇÃO}

O internamento hospitalar de jovens ou idosos são considerados de grande risco, principalmente para os pacientes que não realizam deambulação. ${ }^{1,2}$ Geralmente, é seguido por uma diminuição da capacidade funcional, e mudanças na qualidade de vida, podendo haver também perda da força muscular, inclusive respiratória, devido ao tempo de imobilização e repouso prolongado no leito. ${ }^{3,4}$

Esse declínio não está relacionado apenas à musculatura esquelética periférica, mas também leva em consideração o quadro de disfunções pulmonares. A fisioterapia tem sido amplamente requisitada, com o intuito reverter ou amenizar tal quadro, evitando o desenvolvimento das complicações e diminuindo o tempo de permanência hospitalar. 5

Alguns meios subjetivos possibilitam estimar esse prejuízo físico em hospitalizados, contudo a dinamometria manual apresenta ampla aplicabilidade, pois é um método de baixo custo, simples, rápido e não-invasivo, que fornece, por meio dos valores de Força de Preensão Palmar (FPP), um indicador da saúde geral dos indivíduos avaliados. $^{7-9}$

As disfunções pulmonares e neuromusculares podem ser detectadas previamente através da mensuração das pressões respiratórias máximas (Pressão
Inspiratória Máxima - PImáx e Pressão Expiratória Máxima - PEmáx), pelo teste de manovacuometria que são capazes de demonstrar a força muscular respiratória.

Diante do exposto houve a necessidade de avaliar o comportamento da força muscular respiratória e força de preensão palmar, relacionando as variáveis ao tempo de internação de pacientes atendidos pela fisioterapia em um hospital geral. O estudo foi realizado devido poucos achados literatura, mostrando assim a importância da fisioterapia no ambiente hospitalar em diversos diagnósticos e em diferentes períodos de internação.

Assim, os objetivos do presente estudo foram caracterizar o perfil dos pacientes internados na Santa Casa de Misericórdia de Presidente Prudente, encaminhados à Fisioterapia e avaliar a Pressão Inspiratória máxima (Pimax), Pressão Expiratória máxima (PEmax) e força de preensão palmar (FPP) considerando o tempo de permanência hospitalar.

\section{MÉTODOS}

Este é um estudo clinico com características prospectivas. Foram analisados dados de 93 pacientes de ambos os sexos e idade superior a 18 anos, internados na Santa Casa de Misericórdia de Presidente Prudente que realizaram fisioterapia . 
Os voluntários foram informados sobre os procedimentos e objetivos deste estudo, e após concordarem, assinaram um termo de consentimento livre e esclarecido. Todos os procedimentos utilizados foram aprovados pelo Comitê de Ética da Santa Casa e pelo Comitê de Ética em Pesquisa da Faculdade de Ciências e Tecnologia FCT/UNESP (Proc. no 02592612.8.0000.5402) obedecendo a Resolução 466/2012 do Conselho Nacional de Saúde de 12/12/2012.

A Estatística descritiva foi composta por valores de média, desvio-padrão, intervalos de confiança de $95 \%$ e taxas (\%). Para os dados categóricos, o teste quiquadrado comparou proporções, ao passo que o teste t-student para dados pareados comparou os valores numéricos entre a admissão e alta do paciente. A correlação de Pearson analisou o relacionamento entre as variáveis numéricas envolvidas no estudo.
Todos os resultados foram discutidos no nível de $5 \%$ de significância. O software utilizado foi o BioEstat em sua versão 5.0.

\section{RESULTADOS}

Diante das análises, verificou-se que o motivo de internação foi variado, prevalecendo como diagnóstico principal o câncer com 31,1\%, seguido de 19,3\% de doentes cardíacos, $10,7 \%$ de doentes respiratórios, $14 \%$ ortopédicos, $10 \%$ renais crônicos e 15\% outras causas de internação.

A Tabela I indica as características gerais da amostra no momento da admissão hospitalar. Oservar que o número de homens e mulheres foi similar $(p=0,604)$, bem como, o número de hipertensos $(p=0,917)$. Foi observada grande parcela de pessoas com dislipidemias e diabetes mellitus. O tempo médio de internação foi de 12,4 dias.

Tabela 1. Características gerais da amostra no momento da admissão hospitalar.

\begin{tabular}{|c|c|c|}
\hline Variáveis categóricas & $\%$ & Teste qui-quadrado \\
\hline Sexo (Masculino / Feminino) & $52,7 / 47,3$ & 0,604 \\
\hline Hipertensão Arterial (Sim / Não) & $49,5 / 50,5$ & 0,917 \\
\hline Diabetes Mellitus (Sim / Não) & $30,1 / 69,9$ & 0,001 \\
\hline Hipercolesterolemia (Sim / Não) & $20,4 / 79,6$ & 0,001 \\
\hline Hipertrigliceridemia (Sim / Não) & $16,1 / 83,9$ & 0,001 \\
\hline Sedentarismo (Sim / Não) & $68,8 / 31,2$ & 0,001 \\
\hline \multicolumn{3}{|l|}{ Variáveis Numéricas } \\
\hline Idade (anos [média \pm DP]) & $62,6 \pm 13,3$ & --- \\
\hline Tempo de internação (dias [média \pm DP]) & $12,4 \pm 10,5$ & --- \\
\hline
\end{tabular}


Quando comparados entre os e esquerda (Diferença média: 0,226 momentos de admissão e liberação [Diferença|c95\%: -0,930; 1,382]). Padrão hospitalar, observou-se que não houve variações significativas para as medidas de pressão manual da mão direita (Diferença média: 0,280 [Diferença।c95\%:0,940; -0,380]) similar foi observado para os valores de $\mathrm{PI}$ (Diferença média: -2,452 [Diferença 1 c95\%: $0,364 ;-5,267])$ e PE (Diferença média: $-2,538$ [Diferença $\left.\left.{ }_{1 \mathrm{c} 95 \%}: 0,046 ;-5,121\right]\right)$.

Tabela 2. Valores de pressão manual entre pacientes no momento da admissão e liberação.

\begin{tabular}{|c|c|c|c|}
\hline \multirow{3}{*}{ Variáveis } & Admissão & Liberação & \multirow{3}{*}{$p$} \\
\hline & & & \\
\hline & Média \pm DP & Média \pm DP & \\
\hline FPM (mão direita) & $28,34 \pm 13,7$ & $28,6 \pm 13,8$ & 0,402 \\
\hline FPM (mão esquerda) & $26,5 \pm 14,1$ & $26,3 \pm 13,8$ & 0,699 \\
\hline $\mathrm{PI}\left(\mathrm{cm} / \mathrm{h}_{2} \mathrm{O}\right)$ & $55,8 \pm 29,8$ & $53,3 \pm 28,5$ & 0,087 \\
\hline $\mathrm{PE}\left(\mathrm{cm} / \mathrm{h}_{2} \mathrm{O}\right)$ & $53,2 \pm 25,1$ & $50,7 \pm 26,1$ & 0,054 \\
\hline
\end{tabular}

FPM= força de preensão manual; $\mathrm{PI}=$ pressão de inspiração; $\mathrm{PE}=$ pressão de expiração; $\mathrm{DP}=$ desvio-padrão.

No intuito de identificar se esta ausência de diferenças foi afetada pelo tempo de internação do paciente, analisouse a correlação entre a variação nos valores de FPM e o tempo de internação. Tanto para a mão direita $(r=0.047 ; p=0,653)$ como para a esquerda $(r=-0.030 ; p=0,775)$, a força de preensão manual não relacionou com 0 tempo de internação. Similarmente, não houve relação significativa também para os valores de PI $(r=-0.091 ; p=0,386)$, por outro lado, houve relação entre tempo de internação e valores de PE $(r=-0.252 ; p=$ $0,015)$. Entre os pacientes analisados, as modificações na PI e PE não foram relacionadas à FPM da mão direita $(r$ variando de 0.04 a 0.14) e esquerda $(r$ variando de -0.12 a 0.09 ).

\section{DISCUSSÃO}

Neste estudo, a casuística analisada apresentou uma elevada ocorrência de doenças endócrinas, metabólicas e cardiovasculares. Uma possível justificativa para este padrão se justifica, não apenas na alta ocorrência de sedentarismo, mas também a idade avançada da amostra. Sabe que o envelhecimento está atrelado a uma maior ocorrência de doenças, bem como que pessoas de idade mais avançada apresentam 
menor prática de atividades físicas na velhice e nos anos anteriores ${ }^{10,11}$

Na casuística analisada não houve variações significativas na força de preensão palmar, pressão inspiratória e expiratória máximas. Estes achados nos levam a postular a importância da fisioterapia no ambiente hospitalar quando se leva em conta o declínio funcional decorrente do repouso prolongado e imobilismo durante o período de internação. Dados similares foram encontrados no estudo de Freitas et al. ${ }^{12}$ (utilizando o teste de manovacuômetria), o qual comparou dois grupos de pacientes internados, com e sem o acompanhamento da fisioterapia, encontrando valores significativamente menores que o previsto tanto para a PImax como para a PEmax, no grupo que não foi acompanhado pela fisioterapia.

Foi possível observar também que os valores da força de preensão palmar encontrados nesta população nas avaliações iniciais e finais se encontravam acima da média para mulheres e abaixo para homens dos EUA, Austrália, Canadá, Ucrânia e Suécia, ambos na mesma faixa de idade,para a população Brasileira estes valores se encontravam abaixo dos valores de referência para homens e próximos aos valores de referência para mulheres na mesma faixa de idade. ${ }^{9}$
Quanto a inexistência de relacionamento entre as modificações da força de preensão palmar e força muscular respiratória destes indivíduos durante o período de internação, vale ressaltar que foram avaliados pacientes com diagnósticos diversos, o que pode ter contribuído para este resultado. Um ensaio clínico controlado avaliou sessenta e quatro indivíduos adultos, com fibrose cística, observando redução da força de quadríceps, do consumo de oxigênio e da distância percorrida no teste de caminhada de seis minutos, sem que observasse alterações na Pimáx ${ }^{13}$. Já no estudo realizado por Soares et al. $^{14}$ foi observada uma associação positiva entre a força dos músculos respiratórios e a força de preensão palmar em pneumopatas. ${ }^{13}$

De acordo com outros estudos descritos na literatura este trabalho demonstra a fisioterapia hospitalar como recurso útil para a manutenção/melhora. Por outro lado, as limitações do estudo precisam ser indicadas. Embora os pacientes tenham sido submetidos à fisioterapia durante a internação, no delineamento de estudo apenas acompanhou estes pacientes e não conduziu a intervenção. Nesse sentido, futuros ensaios clínicos são necessários para identificar claramente o método de intervenção mais apropriado. 


\section{CONCLUSÃO}

Os resultados obtidos apontam um perfil heterogêneo de pacientes, com média de idade avançada e diagnósticos diversos, sendo o câncer mais incidente. Além disto, podemos concluir que a força muscular respiratória e a preensão palmar não foram diminuídas pelo período de internação nesses pacientes, no qual realizaram a fisioterapia durante o período de internação, confirmando sua eficácia em pacientes hospitalizados, por prevenir os efeitos deletérios decorrente do repouso prolongado e da inatividade física.

\section{REFERÊNCIAS}

1. REIS, J.S et al. Caracterização da Força Muscular Respiratória e da Capacidade Funcional de Pacientes Internados em uma Enfermaria. Rev Fisioter $S$ Fun. Fortaleza 2012; 1(2): 3-9.

2. KORTEBEIN, $P$ et al. Effect of 10 Days of Bed Rest on Skeletal Muscle in Healthy Older Adults. JAMA 2007; 297(16): 1772-4. http://dx.doi.org/10.1001/jama.297.16.1772$\underline{b}$

3. OVANDO, L.; KOOPMANM; COUTO. Atividades psicomotoras como intervenção no desempenho funcional de idosos hospitalizados. Rev. O Mundo da Saúde, 2010;34(2):176-82.

4. PAIVA, L.C. Úlcera de Pressão em Pacientes Internados em um Hospital Universitário Em Natal/Rn:Condições Predisponentes e Fatores de Risco.[Tese mestrado] CDU 616083:519.873.
5.

ALI, N.A et al. Acquired Weakness, Handgrip Strength, and Mortality in Critically III Patients. Am J Respir Crit Care Med. 2008; 178: 261-8. http://dx.doi.org/10.1164/rccm.200712$18290 \mathrm{C}$

6. NOVAES, R.D et al. Equações de referência para a predição da força de preensão manual em brasileiros de meia idade e idosos. Fisioter Pesq. 2009; 16(3): 217-22. http://dx.doi.org/10.1590/S180929502009000300005

7. SCHUSSEL, M.M.; ANJOS, L.A.; KAC, G. A dinamometria manual e seu uso na avaliação nutricional. Rev. Nutr., 2008; 21(2):223-235.

8. CARVALHO, E.M et al. Força muscular e mortalidade na lista de espera de transplante de fígado. Ver. Bras. Fisioter. 2008; 12(3): 235-40.

http://dx.doi.org/10.1590/S141335552008000300012

9. NASCIMENTO, M.F et al. Valores de referência de força de preensão palmar manual em ambos os gêneros e diferentes grupos etários. Um estudo de revisão.EFDesportes 2010; 15(151).

10 V Diretrizes Brasileiras de Hipertensão Arterial. São Paulo: Sociedade Brasileira de Hipertensão; 2006.

11. FERNANDES, R.A.; ZANESCO, A. Early physical activity promotes lower prevalence of chronic diseases in adulthood. Hypertens Res. 2010; 33(8):926-31. http://dx.doi.org/10.1038/hr.2010.106

12.

FREITAS, E.R.F.S et al. Consequências do estado nutricional na força muscular respiratória de idosos hospitalizados assistidos pela fisioterapia. ASSOBRAFIR Ciência 2009; 69-79. 
13. TROOSTERS, $T$ et al. Pulmonary rehabilitation in chronic obstructive pulmonary disease. Am J Respir Crit Care Med. 2005 Jul 1;172(1):19-38. http://dx.doi.org/10.1164/rccm.2004081109SO

14. SOARES, C.E.N et al. Avaliação muscular respiratória e de membros superiores em pneumopatas. Assobrafir Ciência 2010 Set;1(1):35-46.

Recebido para publicação em 11/08/2014

Revisado em 27/08/2014

Aceito em 01/09/2014 